\title{
Identification of Proteins Interacting with Ubiquitin Chains
}

\author{
Xiaohui Zhao ${ }^{+}$, Joachim Lutz, Eva Höllmüller, Martin Scheffner,* Andreas Marx,* and \\ Florian Stengel*
}

\begin{abstract}
Ubiquitylation, the modification of proteins with ubiquitin $(U b)$, is one of the most versatile post-translational modifications in eukaryotic cells. Since Ub also serves as its own substrate, proteins can be modified by numerous different Ub chains, in which the individual moieties are linked via one or several of the seven lysines of Ub. Homogeneous Ub chains, in which the moieties are sequentially linked via the same residue, have been most extensively studied. However, due to their restricted availability, the functions of Ub chains linked via K27, K29, or K33 are poorly understood. We have developed an approach that, for the first time, allows the generation of all seven homogeneous $\mathrm{Ub}$ chains in large quantities. The potential of our approach is demonstrated by the identification of previously unknown interaction partners of K27-, K29-, and K33-linked Ub chains by affinity-based proteomics.
\end{abstract}

Tagging proteins with ubiquitin (Ub), a highly conserved 76amino acid polypeptide, is one of the most prevalent and versatile post-translational modifications in eukaryotes. Many cellular processes are regulated by ubiquitylation, including protein degradation, cell division, and intracellular signaling. ${ }^{[1]}$ Accordingly, deregulation of ubiquitylation has been found to be causative for various human diseases, such as cancer and neurodegeneration. ${ }^{[2]}$

Ubiquitylation is mediated by a complex enzymatic cascade and results in the formation of different conjugates. In its most simple form, one $\mathrm{Ub}$ moiety is attached to substrate proteins through the formation of an isopeptide bond between the carboxylate of the $\mathrm{C}$-terminal glycine of $\mathrm{Ub}$ and the $\mathrm{E}-\mathrm{NH}_{2}$-group of a lysine in the substrate. ${ }^{[1]}$ Ub contains seven lysines (K6, K11, K27, K29, K33, K48, K63) and each of these, as well as the $\mathrm{N}$-terminal $\alpha-\mathrm{NH}_{2}$ group, can be ubiquitylated. Thus, substrate proteins can be modified with $\mathrm{K}$-linked Ub chains (i.e., Ub moieties are connected via lysine residues by isopeptide bond formation) or in some cases with linear chains (i.e., Ub moieties are linked via the $\alpha-\mathrm{NH}_{2}$ group by conventional peptide bonds). ${ }^{[3]}$ Moreover, various Klinked chain types exist, in which Ub moieties are linked via

$\left[{ }^{*}\right]$ Dr. X. Zhao, ${ }^{[+]}$J. Lutz, ${ }^{[+]}$E. Höllmüller, Prof. Dr. M. Scheffner, Prof. Dr. A. Marx, Prof. Dr. F. Stengel Departments of Chemistry and Biology, Konstanz Research School Chemical Biology, University of Konstanz Universitätsstrasse 10, 78457 Konstanz (Germany)

E-mail: martin.scheffner@uni-konstanz.de andreas.marx@uni-konstanz.de florian.stengel@uni-konstanz.de

$\left.{ }^{+}\right]$These authors contributed equally to this work. the same (homogeneous chains), different (heterogeneous chains), or two or more (branched chains) lysine residues per Ub moiety. ${ }^{[3]}$ The theoretically infinite number of different chains and the notion that different $\mathrm{Ub}$ chains may direct modified proteins to different fates make ubiquitylation one of the most complex and versatile regulators of biological processes.

Homogeneous Ub chains are the most extensively studied chain types. K48-linked chains, and presumably K11-linked chains, target substrates for proteasomal degradation, while K63-linked and linear chains regulate the function of proteins in a non-proteolytic manner, for example, in signal transduction pathways and DNA repair. ${ }^{[4]}$ The physiological functions of other chain types (e.g. K27, K29, K33) are less well understood, which is in part due to the fact that only very few linkage-selective interaction partners are known so far. ${ }^{[5]}$

A difficulty in studying K-linked Ub chains is to obtain these in sufficient amounts and lengths. For instance, the enzymatic assembly of K27-linked chains is still not feasible due to a lack of corresponding conjugating enzymes. ${ }^{[6]}$ Much effort has been made to synthesize Ub chains through protein polymerization. Brik and co-workers have utilized solidphase peptide synthesis combined with native chemical ligation to assemble K48- or K63-linked chains. ${ }^{[7]}$ Strieter and co-workers have employed thiol-ene coupling to synthesize homogeneous $\mathrm{Ub}$ chains linked at $\mathrm{K} 6$, K11 or K63. ${ }^{[8]}$ These isopeptide-linked or thioether-linked Ub polymers were utilized to study the selectivity of de-ubiquitylating enzymes (DUBs) for distinct Ub-Ub linkages. However, since they can be hydrolyzed by DUBs, and since eukaryotic cells are rich in DUBs, the applicability of these Ub chains for the identification of chain-selective interaction partners is limited. ${ }^{[9]}$ Therefore, we and others have developed approaches to generate DUB-resistant triazole-linked chains and reported the synthesis of four linkage-specific chains (K11, K27, K29, K48). ${ }^{[10]}$ However, expression and purification of the monomer precursors were cumbersome, since two unnatural amino acids (UAAs) had to be incorporated into the same Ub in a cell-based system, which not only lowered the yields of full-length Ub but also resulted in the formation of truncated forms of Ub. Stanley and Virdee, and Brik and co-workers have described a similar concept for the generation of K6-linked Ub chains and Ub-protein conjugates, respectively, through oxime ligation. ${ }^{[11]}$ However, so far none of the developed methods has been shown to enable the generation of all seven homogeneous chains in sufficient yields or the discovery of chain-selective interaction partners.

Here, we present a facile and robust approach for the preparation of all seven Ub chains (up to ten Ub moieties) by isopeptide bond mimicry in milligram quantities. The approach combines the incorporation of a single UAA into 
Ub by selective pressure incorporation (SPI) with Michael addition and copper(I)-catalyzed azide-alkyne cycloaddition (CuAAC) reactions. By using such DUB-resistant polymers in a mass spectrometry (MS)-based affinity enrichment assay, we identified several proteins that interact with K27-, K29-, and/or K33-linked chains, thus demonstrating the potential of our approach to elucidate the physiological functions of $\mathrm{Ub}$ chains in general and of so far poorly characterized Ub chains in particular.

To functionalize $\mathrm{Ub}$ with an azide at its C-terminus, we constructed a Ub cDNA in which the Gly76 codon was deleted and Gly 75 was replaced with a codon for methionine to enable the incorporation of azidohomoalanine (Aha) by SPI (Figure 1A). ${ }^{[12]}$ In this way, the later generated triazole linkage has a similar number of atoms as the native isopeptide linkage. To avoid incorporation of Aha at the start codon of $\mathrm{Ub}$ (note that wild-type Ub does not harbor any methionine except for the initial one), a fusion protein system was used consisting of $\mathrm{Ub}$ lacking the initial Met, fused to the C terminus of glutathione-S-transferase (GST). ${ }^{[12]}$ For purification, a thrombin cleavage site was inserted between GST and Ub. To equip the azide-functionalized Ub (Aha75Ub) with an alkyne group, the Ub cDNA was mutated such that the lysine at the desired linkage site is replaced with cysteine
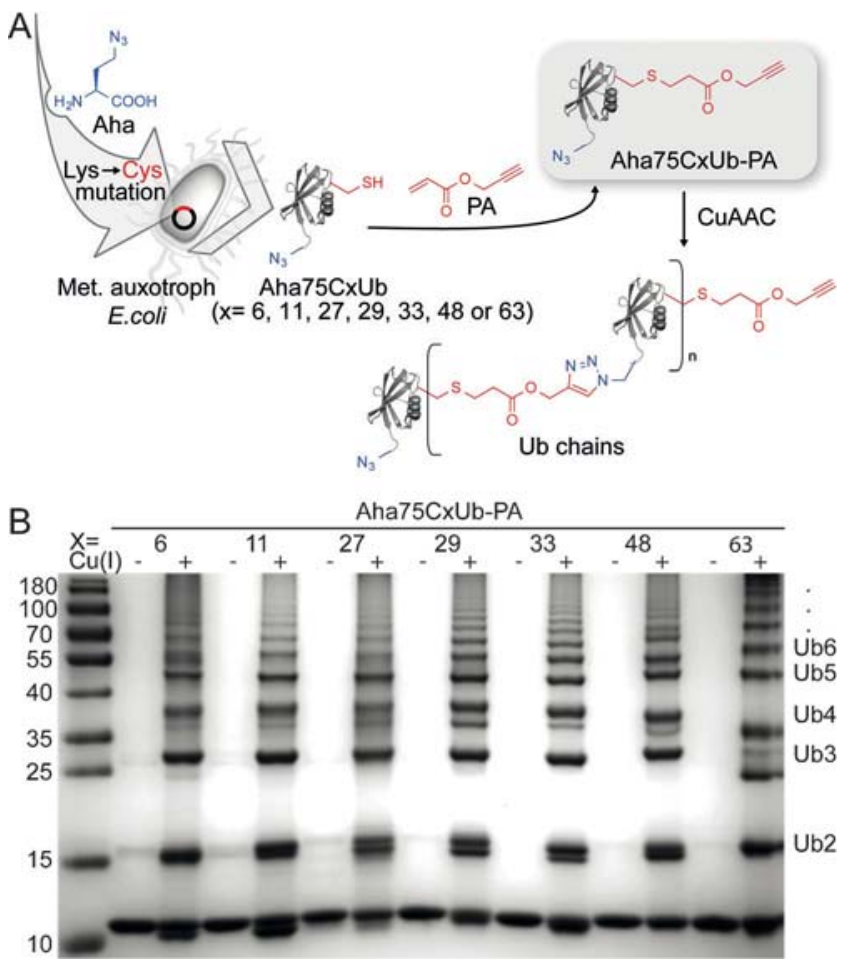

Figure 1. Synthesis of homogeneous Ub chains. A) Schematic of Ub chain preparation, including incorporation of azidohomoalanine (Aha) into $U b$ in E. coli, bioorthogonal modification of Ub with propargyl acrylate (PA), and protein polymerization by copper(I)-catalyzed azidealkyne cycloaddition (CuAAC). B) The resulting chains were resolved by SDS-PAGE followed by staining with Coomassie blue. Note the appearance of doublet bands for some linkages, especially at the position of dimers and tetramers. The faster migrating, less intense bands likely represent cyclized Ub oligomers (Figure S3). The correct position of the linkage sites was validated by MS analysis (Figure S4).
(Aha75CxUb), which can be functionalized through Michael addition with propargyl acrylate (PA; Figure 1A; wild-type $\mathrm{Ub}$ does not harbor any cysteine). In this way, seven Ub monomers Aha75C $x \mathrm{Ub}(x=6,11,27,29,33,48$, or 63$)$ were generated by methods we previously established (Figures S1, S2 in the Supporting Information) and used for CuAACmediated protein polymerization. ${ }^{[12]}$ This resulted in the efficient assembly of all seven K-linked Ub chains with significant amounts of longer Ub oligomers (Figure 1B).

Next, we developed an affinity enrichment approach for the identification of chain-selective interacting partners (Figure 2A). We focused on K27-, K29-, and K33-linked Ub chains because their physiological functions are poorly characterized so far. To do so, we labeled these chains with azide-desthiobiotin, which enables their purification through affinity chromatography. Then, the three different chain types, as well as the Ub monomer (mono-Ub), were immobilized as an affinity matrix on streptavidin-conjugated agarose. "Blank" streptavidin-conjugated agarose beads were used as an additional control. After incubation with HEK293T cell lysate (note that the triazole-linked Ub chains cannot be hydrolyzed by DUBs and thus stay intact in cell lysate; Figure S5) and thorough washing, the Ub polymers and proteins bound to these were eluted with biotin. The elution fractions were resolved by SDS-PAGE (Figure S6) and subsequently analyzed by LC-MS/MS and labelfree quantification. ${ }^{[13]}$

Since our aim was to identify interaction partners of the three different Ub chains, significant enrichment of potential binding proteins was determined with respect to proteins found in the mono-Ub sample (Figures 2B-D; for a full list see Table S1). The Venn diagram in Figure 2E provides an overview on how many of the enriched proteins were specific for one linkage type and how many of these were found to interact with different linkage types. For K27-linked Ub chains, we were able to identify 70 interaction partners. For K29- and K33-linked chains, we identified 44 and 37 proteins, respectively. A majority of $72.8 \%$ (51 out of 70 ) of the interaction partners with K27-linked chains were unique for this linkage type. For chains linked via K29 and K33, the selectivity was lower: $31.8 \%$ (14 out of 44 ) and $24.3 \%$ (9 out of 37), respectively. K29- and K33-linked chains also showed a relative strong overlap in interaction partners (24 out of 44 for K29-linked chains and 24 out of 37 for K33-linked chains), thus suggesting similar biological functions. Importantly, the proteins that were selectively enriched in the mono-Ub samples showed a high degree of consistency for all 3 investigated linkage types (Table S1), thus corroborating the reproducibility and robustness of our approach.

Next, we confirmed the interaction of some of the identified proteins with the respective chain type by western blot analysis (Figure 3). The obtained results were in excellent agreement with our proteomic profiling data. Of special interest were the proteins identified as selective interaction partners of K27-linked Ub chains, since this linkage type has the least information available among the homogeneously linked chains. We confirmed the interactions by western blot analysis for all three tested proteins: PLAA, RANBP9 and AIFM1 (Figure 3). 

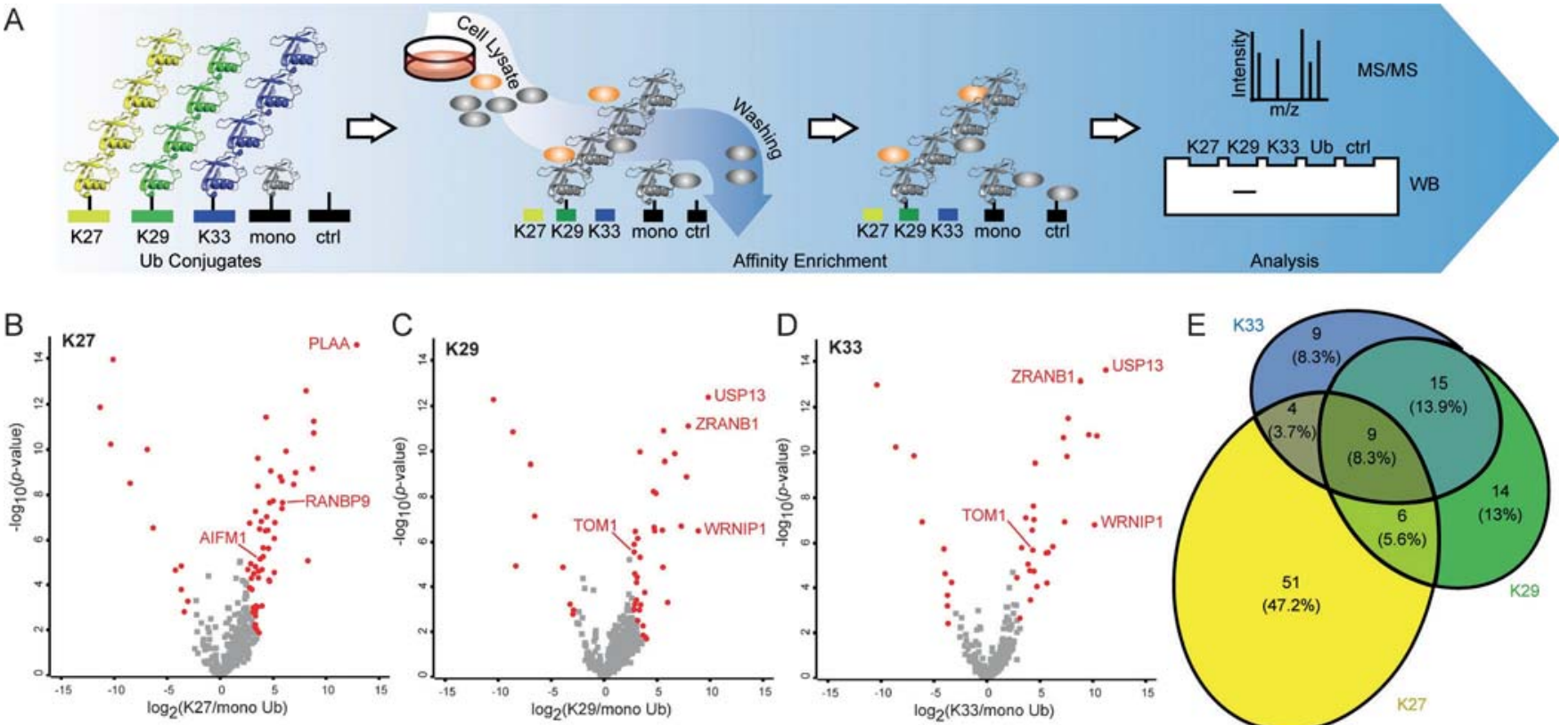

Figure 2. Identification of Ub-chain interaction partners through affinity-based proteomic profiling. A) Schematic of the workflow. K27-, K29-, and K33-linked Ub chains and monomeric Ub (mono) were used as affinity matrices with streptavidin-conjugated agarose beads as a control. Affinity purifications with the different Ub chains and controls were performed in parallel and with four biological replicates. B-D) Volcano plots of proteins identified with K27-, K29-, and K33-linked Ub chains by LC MS/MS and label-free quantification. Significant enrichment was determined relative to proteins found in the mono Ub sample. Plotted is the $\log _{2}$ fold change versus the negative logarithm of the $\mathrm{p}$-values. $\log _{2}=0$ indicates no enrichment compared to the mono Ub fraction. Red dots indicate significant enrichment (FDR $=0.01, \mathrm{~S} 0=2$, two sample t-test). Labeled proteins were selected for confirmation through western blot analysis. E) Overview on the shared and linkage-selective interacting proteins identified for each chain type.

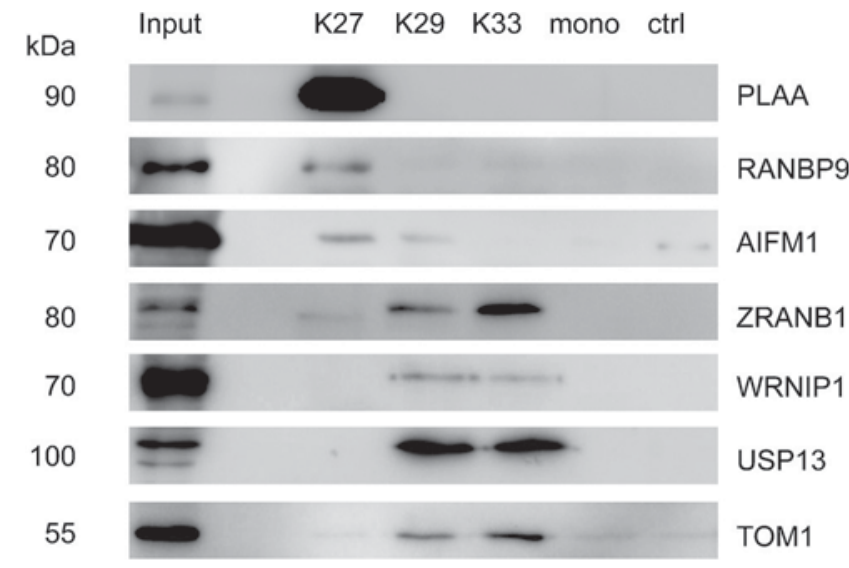

Figure 3. Confirmation of interactions by western blot analysis. Elution fractions of the affinity enrichment assays were subjected to western blot analysis with specific antibodies for the respective protein. Input represents $0.1 \%$ of the HEK293T cell lysate used in affinity enrichment assays.

The yeast orthologue of PLAA (Ufd3/Doa1) harbors a Ub-binding domain (Pfu domain) and interacts with $\mathrm{Cdc} 48$ (mammalian p97/VCP), an ATPase involved in several Ubdependent pathways. ${ }^{[14]}$ Mammalian PLAA is less well studied, but like Doa1 it has been connected to the endolysosomal system. ${ }^{[15]}$ Gid1, the yeast homologue of RANBP9, is part of a conserved E3 ligase complex involved in the degradation of gluconeogenic enzymes. ${ }^{[16]}$ Whether this is also the case for RANBP9 remains to be shown. AIFM1 is mainly involved in mitochondrial processes, including induction of apoptosis. ${ }^{[17]}$ Notably, K27-linked Ub chains have already been linked to mitochondrial processes. ${ }^{[18]}$

We also confirmed the binding of ZRANB1, WRNIP1, USP13, and TOM1, which according to our MS measurements selectively interact with K29- and K33-linked chains, by western blot analysis (Figure 3). The DUB ZRANB1 is the only so far known interaction partner for K29- and K33linked chains, ${ }^{[19]}$ while WRNIP1 is a DNA repair protein containing a UBZ domain that binds to Ub chains. ${ }^{[20]}$ The DUB USP13 has been shown to be involved in various processes such as autophagy and endoplasmic reticulum associated degradation (ERAD). ${ }^{[21]}$ TOM1 has been suggested to be involved in the endosomal trafficking of ubiquitylated proteins, but no preference for a certain linkage type has been reported do date. ${ }^{[22]}$

Finally, the interaction of ZRANB1, WRNIP, USP13, and TOM1 with K29- and K33-linked chains was confirmed by competition experiments using the respective isopeptidelinked Ub tetramers (Figure S7). Indeed, addition of K29and K33-linked Ub tetramers efficiently competed with the binding of ZRANB1, WRNIP, USP13, and TOM1 to the respective triazole-linked Ub chains, while binding of PLAA to K27-linked chains was not affected. This not only shows that the proteins identified bind to the Ub chains studied in a linkage-selective manner (i.e. K29- and K33-linked chains cannot compete with K27-linked chains for PLAA binding, while ZRANB1, WRNIP, USP13, and TOM1 are bound by 
K29- and K33-linked chains but not by K27-linked chains) but also strongly indicates that the triazole-linked $\mathrm{Ub}$ chains resemble native isopeptide-linked $\mathrm{Ub}$ chains at the structural and functional level.

Recently, Zhang et al. published a comprehensive study on the interaction partners of Ub dimers, in which the Ub moieties were connected by a triazole linkage that is structurally different to the one introduced here. ${ }^{[23]}$ Comparison of the data shows that five out of the seven proteins (PLAA, ZRANB1, WRNIP1, USP13, TOM1) studied here were also identified by Zhang et al., with some of these (PLAA, WRNIP1, USP13) not showing much preference in their binding behavior to differently linked Ub dimers. Although we identified them as interaction partners that discriminate between K27-linked (PLAA) and K29- and K33linked (WRNIP1, USP13) Ub chains, they may in addition have the potential to bind to Ub chains linked via residues other than K27 or K29/K33. Furthermore, two proteins identified here as interaction partners of K27-linked chains (RANBP9, AIFM1) were not identified by Zhang et al. at all. Since Ub polymers longer than Ub dimers were employed in our study, this data supports the notion that linkage-selective protein interactions are affected by the length of the Ub chains. ${ }^{[24]}$ Our approach for generating linkage-specific Ub chains thus significantly expands the toolbox for studying the interaction of proteins with Ub chains in a linkage-selective manner and paves the way to a more detailed understanding of the interaction landscape of linkage-defined Ub chains.

In conclusion, we present a robust method for the generation of all seven homogeneous K-linked Ub chains. The chains bear a significant degree of polymerization (up to $10 \mathrm{Ub}$ moieties) and are stable in cell lysates. This enabled us to identify interaction partners of the three poorly characterized linkage types K27, K29, and K33 through affinitybased proteomic profiling. Importantly, several of the interactions identified by MS were confirmed by western blot analysis, thus demonstrating the potential of our approach. The presented method provides an important tool to unravel the complex mechanisms of Ub signaling.

\section{Acknowledgements}

This work was supported by the DFG (SFB969, project B3). X.Z. and E.H. thank the DAAD and the Konstanz Research School Chemical Biology, respectively, for support by a fellowship. F.S. acknowledges funding from the DFG Emmy Noether Program (STE 2517/1-1). We further thank Dr. Samra Ludmann for graphical support.

\section{Conflict of interest}

The authors declare no conflict of interest.

Keywords: affinity-based profiling · click chemistry . post-translational modification · proteomic profiling . ubiquitylation
[1] a) A. Hershko, A. Ciechanover, Annu. Rev. Biochem. 1998, 67, 425 -479; b) D. Komander, M. Rape, Annu. Rev. Biochem. 2012, $81,203-229$.

[2] D. Popovic, D. Vucic, I. Dikic, Nat. Med. 2014, 20, 1242-1253.

[3] a) M. Hochstrasser, Nature 2009, 458, 422-429; b) R. Yau, M. Rape, Nat. Cell Biol. 2016, 18, 579-586.

[4] a) Y. Kravtsova-Ivantsiv, T. Sommer, A. Ciechanover, Angew. Chem. Int. Ed. 2013, 52, $192-198$; Angew. Chem. 2013, 125, $202-$ 209 ; b) H. D. Ulrich, H. Walden, Nat. Rev. Mol. Cell Biol. 2010, 11, 479-489; c) E. Rieser, S. M. Cordier, H. Walczak, Trends Biochem. Sci. 2013, 38, 94-102.

[5] a) Y. Kulathu, D. Komander, Nat. Rev. Mol. Cell Biol. 2012, 13, 508-523; b) C. Alfano, S. Faggiano, A. Pastore, Trends Biochem. Sci. 2016, 41, 371-385; c) D. Scott, N. J. Oldham, J. Strachan, M. S. Searle, R. Layfield, Proteomics 2015, 15, 844-861.

[6] S. Faggiano, C. Alfano, A. Pastore, Anal. Biochem. 2016, 492, $82-90$.

[7] a) T. Moyal, S. N. Bavikar, S. V. Karthikeyan, H. P. Hemantha, A. Brik, J. Am. Chem. Soc. 2012, 134, 16085-16092; b) K. S. A. Kumar, L. Spasser, L. A. Erlich, S. N. Bavikar, A. Brik, Angew. Chem. Int. Ed. 2010, 49, 9126-9131; Angew. Chem. 2010, 122, $9312-9317$; c) K. S. A. Kumar, S. N. Bavikar, L. Spasser, T. Moyal, S. Ohayon, A. Brik, Angew. Chem. Int. Ed. 2011, 50, 6137-6141; Angew. Chem. 2011, 123, 6261-6265.

[8] a) V. H. Trang, E. M. Valkevich, S. Minami, Y.-C. Chen, Y. Ge, E. R. Strieter, Angew. Chem. Int. Ed. 2012, 51, 13085-13088; Angew. Chem. 2012, 124, 13262-13265; b) E. M. Valkevich, R. G. Guenette, N. A. Sanchez, Y.-C. Chen, Y. Ge, E. R. Strieter, J. Am. Chem. Soc. 2012, 134, 6916-6919; c) V. H. Trang, M. L. Rodgers, K. J. Boyle, A. A. Hoskins, E. R. Strieter, ChemBioChem 2014, 15, 1563-1568.

[9] S. M. Mali, S. K. Singh, E. Eid, A. Brik, J. Am. Chem. Soc. 2017, 139, $4971-4986$.

[10] a) T. Schneider, D. Schneider, D. Rösner, S. Malhotra, F. Mortensen, T. U. Mayer, M. Scheffner, A. Marx, Angew. Chem. Int. Ed. 2014, 53, 12925-12929; Angew. Chem. 2014, 126, 13139-13143; b) S. Eger, M. Scheffner, A. Marx, M. Rubini, J. Am. Chem. Soc. 2010, 132, 16337-16339; c) D. Rösner, T. Schneider, D. Schneider, M. Scheffner, A. Marx, Nat. Protoc. 2015, 10, 1594-1611; d) D. Schneider, T. Schneider, J. Aschenbrenner, F. Mortensen, M. Scheffner, A. Marx, Bioorg. Med. Chem. 2016, 24, 995 -1001; e) N. D. Weikart, H. D. Mootz, ChemBioChem 2010, 11, 774-777; f) S. Sommer, N. D. Weikart, A. Brockmeyer, P. Janning, H. D. Mootz, Angew. Chem. Int. Ed. 2011, 50, 9888-9892; Angew. Chem. 2011, 123, 10062-10066.

[11] a) M. Stanley, S. Virdee, ChemBioChem 2016, 17, 1472-1480; b) S. K. Singh, I. Sahu, S. M. Mali, H. P. Hemantha, O. Kleifeld, M. H. Glickman, A. Brik, J. Am. Chem. Soc. 2016, 138, $16004-$ 16015.

[12] a) M. Scheffner, J. M. Huibregtse, R. D. Vierstra, P. M. Howley, Cell 1993, 75, 495-505; b) D. Schneider, T. Schneider, D. Rösner, M. Scheffner, A. Marx, Bioorg. Med. Chem. 2013, 21, $3430-3435$.

[13] a) J. Cox, M. Y. Hein, C. A. Luber, I. Paron, N. Nagaraj, M. Mann, Mol. Cell. Proteomics 2014, 13, 2513-2526; b) J. Cox, M. Mann, Nat. Biotechnol. 2008, 26, 1367-1372; c) S. Tyanova, T. Temu, P. Sinitcyn, A. Carlson, M. Y. Hein, T. Geiger, M. Mann, J. Cox, Nat. Methods 2016, 13, $731-740$.

[14] a) J. E. Mullally, T. Chernova, K. D. Wilkinson, Mol. Cell. Biol. 2006, 26, 822-830; b) D. Barthelme, R. T. Sauer, J. Mol. Biol. 2016, 428, $1861-1869$.

[15] a) J. Ren, N. Pashkova, S. Winistorfer, R. C. Piper, J. Biol. Chem. 2008, 283, 21599-21611; b) C. Papadopoulos, P. Kirchner, M. Bug, D. Grum, L. Koerver, N. Schulze, R. Poehler, A. Dressler, S. 
Fengler, K. Arhzaouy, V. Lux, M. Ehrmann, C. C. Weihl, H. Meyer, EMBO J. 2017, 36, 135-150.

[16] R. Menssen, J. Schweiggert, J. Schreiner, D. Kušević, J. Reuther, B. Braun, D. H. Wolf, J. Biol. Chem. 2012, 287, 25602-25614.

[17] N. Joza, J. A. Pospisilik, E. Hangen, T. Hanada, N. Modjtahedi, J. M. Penninger, G. Kroemer, Ann. N. Y. Acad. Sci. 2009, 1171, $2-11$.

[18] S. Geisler, K. M. Holmstrom, D. Skujat, F. C. Fiesel, O. C. Rothfuss, P. J. Kahle, W. Springer, Nat. Cell Biol. 2010, 12, 119 131.

[19] a) S. Virdee, Y. Ye, D. P. Nguyen, D. Komander, J. W. Chin, Nat. Chem. Biol. 2010, 6, 750-757; b) J. D. F. Licchesi, J. Mieszczanek, T. E. T. Mevissen, T. J. Rutherford, M. Akutsu, S. Virdee, F. E. Oualid, J. W. Chin, H. Ovaa, M. Bienz, D. Komander, Nat. Struct. Mol. Biol. 2012, 19, 62-71.

[20] R. A. Bish, M. P. Myers, J. Biol. Chem. 2007, 282, 23184-23193.

[21] a) J. Liu, H. Xia, M. Kim, L. Xu, Y. Li, L. Zhang, Y. Cai, H. V. Norberg, T. Zhang, T. Furuya, M. Jin, Z. Zhu, H. Wang, J. Yu, Y. Li, Y. Hao, A. Choi, H. Ke, D. Ma, J. Yuan, Cell 2011, 147, $223-$
234; b) Y. Liu, N. Soetandyo, J.-G. Lee, L. Liu, Y. Xu, W. M. Clemons, Jr., Y. Ye, eLife 2014, 3, e01369.

[22] Y. Katoh, Y. Shiba, H. Mitsuhashi, Y. Yanagida, H. Takatsu, K. Nakayama, J. Biol. Chem. 2004, 279, 24435-24443.

[23] X. Zhang, A. H. Smits, G. B. A. van Tilburg, P. W. T. C. Jansen, M. M. Makowski, H. Ovaa, M. Vermeulen, Mol. Cell 2017, 65, 941-955.

[24] a) D. Fushman, K. D. Wilkinson, F1000 Biol. Rep. 2011, 3, 1-10; b) F. E. Reyes-Turcu, J. R. Shanks, D. Komander, K. D. Wilkinson, J. Biol. Chem. 2008, 283, 19581-19592; c) D. Zhang, T. Chen, I. Ziv, R. Rosenzweig, Y. Matiuhin, V. Bronner, M. H. Glickman, D. Fushman, Mol. Cell 2009, 36, 1018-1033. 